Aim of the study: The available data on serum leptin levels in ovarian cancer present contradictory results. The majority of authors report lower leptin levels in those patients in comparison to healthy individuals. However, there is no data regarding leptin concentrations during therapy in women with primary epithelial ovarian cancer.

Material and methods: Blood samples were collected at the time of diagnosis, after initial surgery, and after first-line chemotherapy. Leptin serum concentrations were analysed using ELISA technique. Additionally, parallel measurements of CA125 levels were performed.

Results: Fifty-three patients with primary epithelial ovarian cancer met the inclusion criteria and were included in our study. Our analysis revealed a significant difference in mean preoperative serum leptin concentrations between early and advanced ovarian cancer patients $(p<0.0001)$. We identified statistically significant elevation of mean serum leptin levels $(p<0.001)$ after complete macroscopic cytoreduction and after first-line chemotherapy in advanced ovarian cancer cases.

Discussion: In this cohort, a significant elevation of postoperative serum leptin levels after complete macroscopic cytoreduction were shown. Moreover, elevation of leptin levels corresponded with remission after chemotherapy. Further studies are needed to determine if leptin can be a potential marker of surgery completeness as well as a marker in chemotherapy response evaluation.

Key words: leptin, ovarian cancer, surgery, chemotherapy.

Contemp Oncol (Pozn) 2014; 18 (5): 318-322 DOI: $10.5114 /$ wo. 2014.46323

\section{Evaluation of leptin serum concentrations during surgery and first-line chemotherapy in primary epithelial ovarian cancer patients}

\author{
Jacek Przemyslaw Grabowski ${ }^{1}$, Anna Markowska ${ }^{2}$, Janina Markowska ${ }^{3}$
}

'Department of Gynaecology and Gynaecological Oncology, Kliniken-Essen-Mitte, Essen, Germany

2Department of Perinatology and Gynaecology, Poznan University of Medical Sciences, Poland

${ }^{3}$ Department of Oncology, Division of Gynaecology, Poznan University of Medical Sciences, Poland

\section{Introduction}

Epithelial ovarian cancer is the fourth leading cause of cancer-related death in women in the United States and the leading cause of gynaecological cancer death [1, 2]. Due to late-onset symptoms, ovarian cancer is mainly diagnosed in an advanced stage and is therefore associated with poor survival [3]. Complete cytoreduction is the single most important prognostic factor for outcome in patients in whom median overall survival reaches 99.1 months [4-6]. In order to achieve these results patients need to be treated in specialised gynaeco-oncological centres. However, there is no objective test, evaluating outcome of surgery. Additionally, monitoring of standard systemic therapy with carboplatin and paclitaxel remains unsatisfactory in some cases.

Zhang et al. initially described leptin in 1994 [7]. It is a product of obesity gene (ob) and is synthesised mainly by white adipose tissue. Nevertheless its expression was detected in other tissues like placenta or cancer cells. Leptin exists in two forms: free and bound, where the free fraction is the active one. It acts as a negative feedback signal critical to normal control of food intake and body weight. Moreover, anti-apoptotic activity in human cells has been reported. Concomitantly to leptin, expression of different isoforms of its receptors was observed in the studied tissues. Para as well as autocrine action pathway of leptin in carcinogenesis remain possible [7, 8].

The available data on leptin serum concentrations in ovarian cancer patients present contradictory results. Tessitore et al. [9] reported higher leptin concentration in ovarian cancer patients. Nevertheless, in most available publications lower leptin concentrations in ovarian cancer patients in comparison to healthy individuals were reported [10-13]. To the authors' knowledge, there are no reports evaluating leptin concentrations during therapy in ovarian cancer patients.

The aim of our study was to evaluate serum leptin levels in ovarian cancer patients before and after primary surgery as well as after first-line chemotherapy. Furthermore, correlations between leptin and CA125, BMI, and FIGO (International Federation of Gynaecology and Obstetrics) stage and grade were analysed.

\section{Material and methods}

Patients with primary epithelial ovarian cancer treated at the Department of Oncology, Division of Gynaecology, Poznan University of Medical Sciences were enrolled in our single-centre study. 
Patients with suspicious ovarian tumours referred to our centre received frozen section examination during primary surgery. In cases with histologically confirmed epithelial ovarian cancer, enrolment was limited to patients who underwent diagnostic procedures (e.g. paracentesis) only. Furthermore, any women with non-epithelial ovarian neoplasms, recurrent disease, other malignancies, and those who received neoadjuvant chemotherapy were disqualified from this study. The control group consisted of patients with benign ovarian findings.

Gynaecological examination, vaginal ultrasound, and additional radiological imaging were standard diagnostic procedures prior to planned surgery. From all patients, weight and height at the time of diagnosis were used to calculate body mass index (BMI).

Informed consent form was obtained from all of the patients in this study, according to the requirements of the Poznan University of Medical Sciences Bioethics Committee.

Fasting serum samples for antibody microarray analysis were prospectively collected from individuals with primary ovarian cancer and from patients with benign ovarian findings. Blood samples were collected three times: at the time of diagnosis, between days 7 and 10 after primary surgery, and during the fourth week after first-line chemotherapy. In a control group, blood samples were taken twice: before and after surgery.

Quantikine Human ELISA assay kits for each of the analytes selected for further analysis were purchased from R\&D Systems. Standard laboratory assay for CA125 concentrations evaluation was used.

Optimal surgery was defined as complete tumour resection and suboptimal as any visible disease residuals. Standard adjuvant chemotherapy consisted of 6 cycles of paclitaxel $175 \mathrm{mg} / \mathrm{m}^{2}$ and carboplatin AUC5 or cisplatin $75 \mathrm{mg} / \mathrm{m}^{2}$ every three weeks.

Statistical analysis was performed using StatSoft Statistica 6.0. Changes of leptin and CA125 concentrations were evaluated using Student $t$-test. Correlation between $\mathrm{BMI}, \mathrm{FIGO}$ stage, grade, and leptin concentrations were evaluated using Spearman test.

\section{Results}

Fifty-three patients with primary epithelial ovarian cancer treated at the Department of Oncology, Division of Gynaecology, Poznan University of Medical Sciences between 2006 and 2007 met the inclusion criteria and were included in our study. Fifty-one women with benign ovarian tumours were included in the control group.

The average age in the control group was 42.5 years (range 29-68), whereas the average age of women in the study group was 53.9 years (range 44-71). All patients were in good performance status ECOG (Eastern Cooperative Oncology Group) 0 or 1.

Fifteen (29\%) women in study group had stage FIGO I/II and 38 (71\%) women had stage FIGO III/IV ovarian cancer. The majority of patients had serous pathology - 39 (73\%), followed by mucinous -7 (13\%), clear cell $-3(6 \%)$, undifferentiated -2 (4\%), and endometrioid - 2 (4\%). The grade was determined in 49 patients, among those 10 (21\%) had G1, 19 (38\%) had G2, and 20 (41\%) had G3.
Thirty-three (63\%) patients in the study group underwent optimal primary surgery with complete tumour resection. Among those, all 15 (100\%) women had early ovarian cancer FIGO I/II and 18 (47\%) patients had advanced ovarian cancer FIGO III.

We observed significantly lower mean serum leptin concentrations in ovarian cancer patients vs. control group, $9.26 \pm 4.04 \mathrm{ng} / \mathrm{ml}$ and $15.25 \pm 2.82 \mathrm{ng} / \mathrm{ml}(p<0.0001)$, respectively (Fig. 1). Patients with advanced ovarian cancer FIGO III/IV had lower mean serum leptin levels in comparison to women with FIGO I/II stage, $7.08 \pm 1.87 \mathrm{ng} / \mathrm{ml}$ and $14.73 \pm 1.87 \mathrm{ng} / \mathrm{ml}$, respectively $(p<0.0001)$ (Fig. 2). No significant differences in mean serum leptin concentrations were observed between patients with early ovarian cancer and the control group. We did not find any correlation between histology or grade and mean serum leptin levels in our study.

Subsequently, postoperative mean leptin concentrations were evaluated. No significant differences between pre- and post-operative mean serum leptin levels in study and control group were identified, $11.04 \pm 4.03 \mathrm{ng} / \mathrm{ml}$ $(p=0.052)$ and $14.9 \pm 3.1 \mathrm{ng} / \mathrm{ml}(p=0.064)$ respectively (Fig. 3). The subgroup analysis showed no significant change of post-operative mean serum leptin concentration in stage FIGO I/II ovarian cancer patients ( $p=0.057$ ). However, comparison of mean pre- and post-operative leptin concentrations in advanced (FIGO III/IV) ovarian

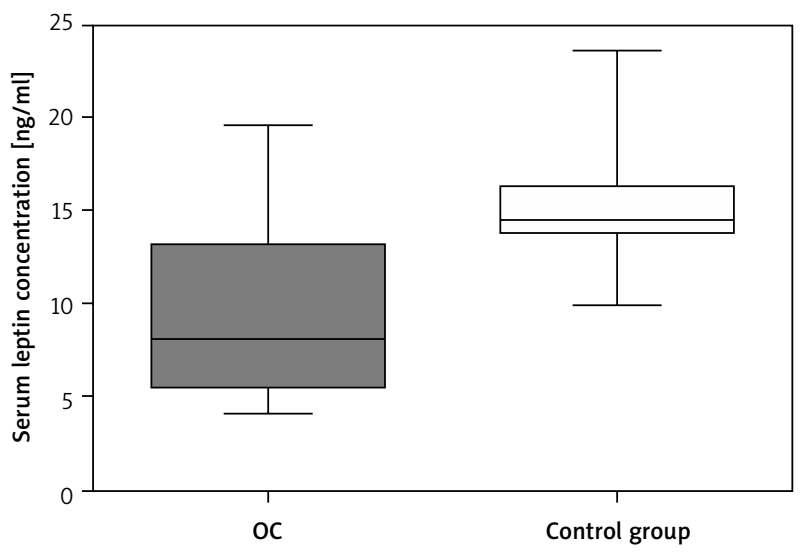

Fig. 1. Mean pre-operative leptin concentrations in ovarian cancer patients (OC) and control group

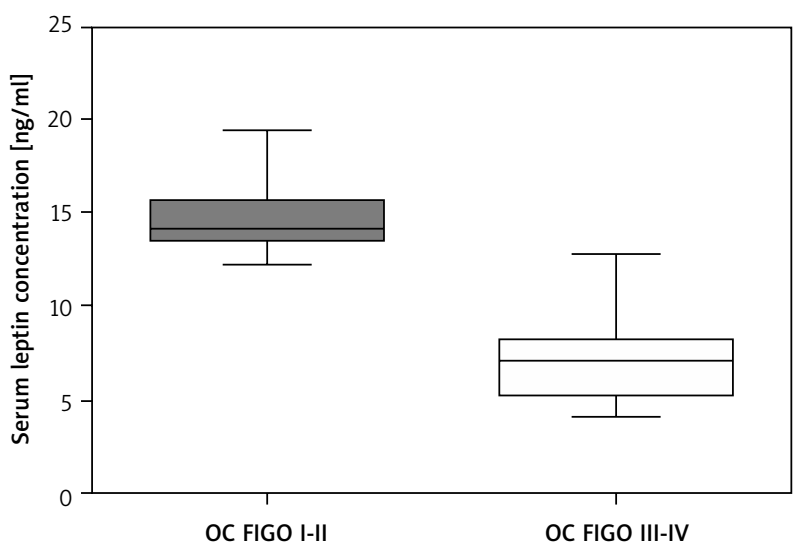

Fig. 2. Mean pre-operative serum leptin concentrations in early FIGO I/II and advanced FIGO III/IV ovarian cancer (OC) patients 


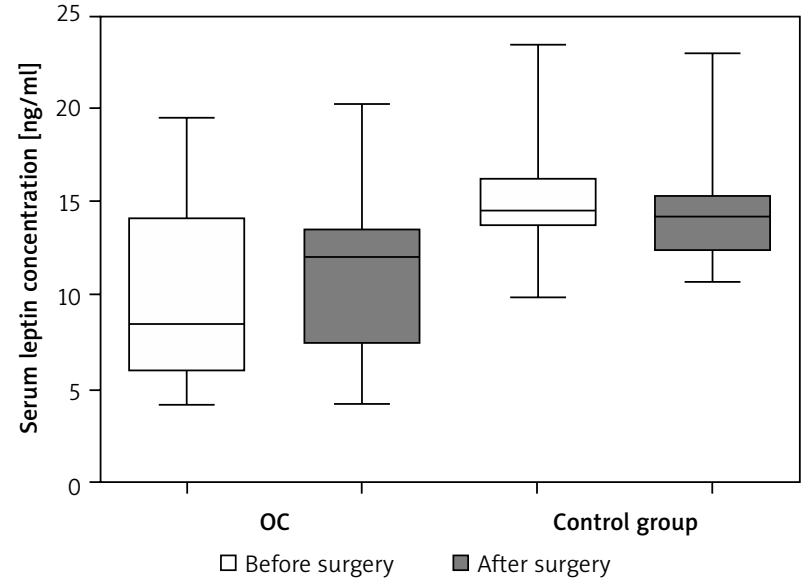

Fig. 3. Pre- and post-operative serum leptin concentrations in ovarian cancer (OC) patients and control group

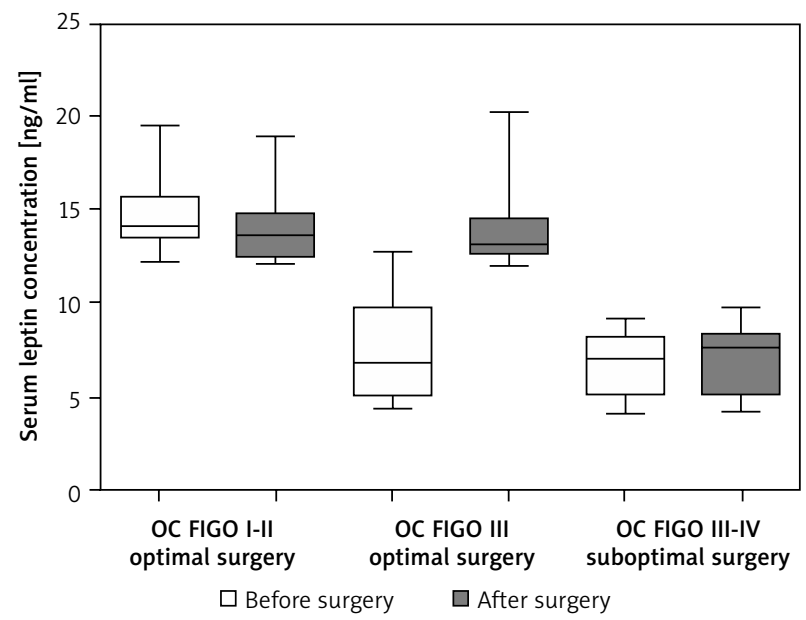

Fig. 4. Mean pre- and post-operative serum leptin concentrations regarding performed surgery and ovarian cancer stage

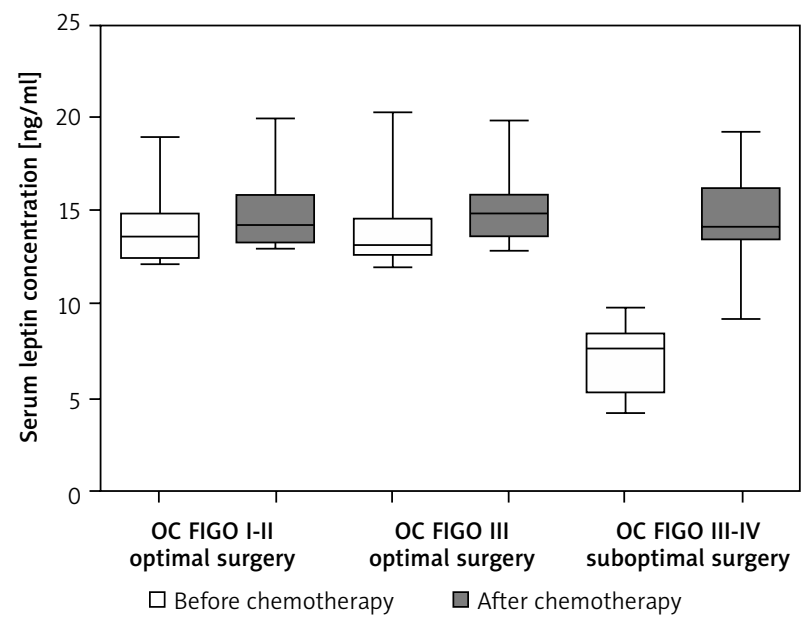

Fig. 5. Comparison of mean serum leptin concentrations before and after first-line chemotherapy

cancer according to performed surgery. Significant elevation to $13.71 \mathrm{ng} / \mathrm{ml}(p<0.001)$ of mean post-operative serum leptin concentrations in patients who underwent complete tumour resection were identified. In contrast, mean post-operative serum leptin levels did not significantly change in patients who underwent suboptimal surgery ( $p=0.059)$ (Fig. 4).

Standard first-line chemotherapy was applied to $52 \mathrm{pa}$ tients in the study group. One patient diagnosed with ovarian cancer FIGO IA G1 did not receive any adjuvant chemotherapy. $94 \%$ of analysed patients received 6 cycles of chemotherapy and the mean number of cycles was 5.8. Significantly higher mean serum leptin concentrations were observed after chemotherapy in comparison to mean post-operative values $-14.82 \pm 4.30 \mathrm{ng} / \mathrm{ml}$ $(p<0.001)$. Strong statistically significant elevation of mean serum leptin concentration in patients who underwent suboptimal cytoreduction was identified in advanced ovarian cancer patients, $14.65 \pm 2.45 \mathrm{ng} / \mathrm{ml}(p<0.001)$. No significant difference was observed in mean serum leptin level after chemotherapy, $14.84 \pm 1.95 \mathrm{ng} / \mathrm{ml}(p=0.054)$, in early ovarian cancer patients in comparison to post-operative mean concentration (Fig. 5).

Mean body mass index (BMI) of patients in the study group was $24 \mathrm{~kg} / \mathrm{m}^{2}$ (range 18.2-32.8) and $22 \mathrm{~kg} / \mathrm{m}^{2}$ (range 17.2-30.3) in the control group ( $p>0.05)$. Mean BMI in patients with early ovarian cancer was $23.7 \mathrm{~kg} / \mathrm{m}^{2}$ (range $18.2-31.9$ ) and in the advanced group $24.4 \mathrm{~kg} / \mathrm{m}^{2}$ (range 19.0-32.8) ( $p>0.05)$. No statistical difference in initial BMI between early, advanced ovarian cancer, and the control group was found ( $p>0.05$ ). We observed positive correlation between serum leptin concentrations and BMI in the control group as well as in early and advanced ovarian cancer patients $(p<0.05)$. However, mean serum leptin concentrations in women with benign ovarian tumours and early ovarian cancer patients (FIGO I/II) were significantly higher in comparison to advanced ovarian cancer patients (FIGO III/IV) by similar BMI values in those groups $(p<0.05)$

Additionally, serum CA125 concentrations in the study group were evaluated. Independently of FIGO stage or surgery, an $86.6 \%$ decrease in post-operative mean serum CA125 level ( $p<0.001)$ was found. The decrease of mean serum CA125 concentration was significantly higher in patients who underwent complete cytoreduction versus sub-optimally operated patients, 92\% (58.8 U/ml) and $82 \%(315.2 \mathrm{U} / \mathrm{ml})(p<0.05)$, respectively. After first-line chemotherapy in patients with advanced disease FIGO III/ IV who underwent complete surgery, mean serum CA125 levels decreased to $28.1 \mathrm{U} / \mathrm{ml}$ (53.2\% decrease) and $34.1 \mathrm{U} /$ $\mathrm{ml}(89.2 \%$ decrease) in women who received suboptimal cytoreduction in comparison to post-operative levels.

\section{Discussion}

The majority of available publications show low serum leptin concentrations in ovarian cancer patients at the time of diagnosis [10-13]. Mor et al. published data of 100 ovarian cancer patients, including 24 FIGO stage I/II cases, in comparison to women with benign adnexal findings [10]. Further investigation by Visintin et al. showed analogical results from 156 women with ovarian malignancies [11]. Moreover, these authors reported differences according to stage of disease. Patients with advanced stage 
disease FIGO III/IV had significantly lower serum leptin levels in comparison to FIGO I/II cases. The results of our research correspond with the above-mentioned studies. Data presented by Mor et al. and Visintin et al., as well as our investigation, are based on the highest number of enrolled patients and presumably reflect the real situation. According to these observations, leptin might be considered as a potential factor in preoperative ovarian-mass diagnostics.

Our study showed statistically significant elevation of serum leptin concentrations after complete cytoreduction in advanced ovarian cancer patients. This correlation was not observed in patients who underwent suboptimal surgery or those with early ovarian cancer. To the authors' knowledge, our study is the first research showing differences in initial and post-operative serum leptin levels. A large number of patients with advanced ovarian cancer present ascites at the time of diagnosis. Abramov et al. reported post-operative elevation of initially low serum leptin concentrations in a patient with Meigs syndrome after removal of ascites [14]. Another study showed low leptin levels in patients with androgen-secreting ovarian tumour and its post-operative normalisation without parallel BMI or fat tissue distribution changes [15]. The above data may suggest a direct association of low serum leptin concentrations with initial tumour burden and/or the presence of ascites. Leptin production depends mainly on body weight, which in our study positively correlated with the BMI of patients. However, we did not find differences in BMI between advanced, early ovarian cancer, and the control group. Barber et al. reported higher resting energy expenditure due to tumour load despite weight loss [16]. Concomitant cancer progression, inflammation, and catabolic processes lead to decrease of serum leptin concentration. Increased energy output induced by tumour growth results in lower leptin production and therefore decreased metabolism and food intake. Nevertheless these processes resulting in decreased leptin serum concentrations begin before loss of weigh appears [17-20]. Several studies showed elevated leptin concentrations associated with cancer risk when its serum levels were measured prospectively [21, 22]. Elevated or normal leptin concentrations were reported in analyses of patients performed 1.5 years before diagnosis for colorectal [22] and breast cancer [22], respectively. However, leptin levels measured at the time of diagnosis in patients with gastrointestinal and other cancers are lower in comparison to control groups $[23,24]$. Therefore, the timing of blood collection in relation to disease onset might significantly affect leptin concentrations. Subsequently, complete removal of the tumour, and therefore normalisation of metabolic processes, might lead to leptin levels elevation. Summarising, the comparison of pre- and post-operative leptin concentrations might be useful in the evaluation of cytoreduction completeness. However, the influence of other factors should not be underestimated and needs further investigation.

A decrease in post-operative serum CA125 levels was also observed in our patients. The change in its concentration was found to be independent in regard to performed surgery. However, in patients who underwent complete cytoreduction, the decrease in post-operative values was significantly greater in comparison to that seen in women who received suboptimal surgery. Furthermore, the latter subgroup of patients presented significantly higher initial CA125 values. Yedema et al. showed post-operative decrease in CA125 in only a minority of investigated patients, concluding the limited role of CA125 in the evaluation of residual tumours [25]. Mogensen et al. reported limited significance of post-operative CA125 levels due to other perioperative factors besides residual disease influencing its concentrations [26]. Nevertheless, in 1996 Gaducci et al. presented differences of pre- and post-operative CA125 levels according to the size of residual tumours [27]. The post-operative decline of CA125 concentrations was significantly higher in patients with smaller residual disease. This observation is in accordance with our study. CA125 in combination with serum leptin levels might serve as a potential marker for cytoreduction completeness monitoring.

Subsequently we analysed leptin concentrations in patients after chemotherapy. According to the findings of the first part of our study, the differences in serum leptin levels varied according to the stage of disease. Highly significant elevation of serum leptin concentrations have been observed in patients with advanced ovarian cancer FIGO III/ IV, who underwent suboptimal primary surgery. In those patients with high postoperative tumour burden, remission achieved due to given chemotherapy resulted in normalisation of leptin concentrations. Comparison to an established ovarian cancer marker CA125 showed a negative correlation between its declining levels and rising leptin concentrations during the chemotherapy. Again, normalisation of leptin levels after completion of therapy might suggest that tumour burden and therefore altered metabolism is responsible for its initial lower concentrations in advanced ovarian cancer cases FIGO III/IV. Nevertheless, the exact mechanism of leptin serum elevation remains unclear and needs further investigation.

Analogically to other authors, we have observed a positive correlation between leptin concentrations and BMI in our patients [28]. However, leptin levels in patients with advanced ovarian cancer were significantly lower in comparison to early ovarian cancer cases and the control group, despite similar mean BMI values. This observation suggests a limited influence of BMI on leptin levels in cancer patients. Association of initially low serum leptin levels with tumour burden or presence of ascites in those cases remains very probable.

The primary complete cytoreduction in ovarian cancer patients remain mainstay in successful treatment of this disease [4-6]. Our study is the first prospective investigation showing differences of serum leptin concentrations in primary ovarian cancer patients during the therapy. Evaluation of pre- and post-operative leptin serum concentrations might be a potential objective tool for surgery completeness monitoring. Moreover, complementary to CA125, it could play a role in chemotherapy response evaluation. Nevertheless, in our opinion further investigations on larger groups of patients remain necessary. 


\section{Acknowledgment}

The Polish Committee for Scientific Research N N407 347533 supported this study.

\section{References}

1. Sant M, Allemani C, Santaquilani M, Knijn A, Marchesi F, Capocac cia R; EUROCARE Working Group. EUROCARE-4. Survival of cancer patients diagnosed in 1995-1999. Results and commentary. Eur J Cancer 2009; 45: 931-91.

2. Jemal A, Siegel R, Ward E, Hao Y, Xu J, Thun MJ. Cancer statistics, 2009. CA Cancer J Clin 2009; 59: 225-49.

3. Permuth-Wey J, Sellers TA. Epidemiology of ovarian cancer. Methods Mol Biol 2009; 472: 413-37.

4. Bristow RE, Tomacruz RS, Armstrong DK, Trimble EL, Montz FJ. Survival effect of maximal cytoreductive surgery for advanced ovarian carcinoma during the platinum era: a meta-analysis. J Clin Oncol 2002; 20: 1248-59.

5. du Bois A, Reuss A, Pujade-Lauraine E, Harter P, Ray-Coquard I, Pfisterer J. Role of surgical outcome as prognostic factor in advanced epithelial ovarian cancer: a combined exploratory analysis of 3 prospectively randomized phase 3 multicenter trials: by the Arbeitsgemeinschaft Gynaekologische Onkologie Studiengruppe Ovarialkarzinom (AGO-OVAR) and the Groupe d'Investigateurs Nationaux Pour les Etudes des Cancers de l'Ovaire (GINECO). Cancer 2009; 115: 1234-44

6. Harter P, Muallem MZ, Buhrmann C, et al. Impact of a structured quality management program on surgical outcome in primary advanced ovarian cancer. Gynecol Oncol 2011; 121: 615-9.

7. Zhang Y, Proenca R, Maffei M, Barone M, Leopold L, Friednam JM. Positionalcloning of the mouse obese gene and its human homologue. Nature 1994; 372: 425-32.

8. Bajari TM, Strasser V, Nimpf J, Schneider J. A model for modulation of leptin activity by association with clusterin. FASEB J 2003; 17: 1505-7.

9. Tessitore L, Vizio B, Jenkins O, De Stefano I, Ritossa C, Argiles JM, Benedetto C, Mussa A. Leptin expression in colorectal and breast cancer patients. Int J Mol Med 2000; 5: 421-6.

10. Mor G, Visintin I, Lai Y, et al. Serum protein markers for early detection of ovarian cancer. Proc Natl Acad Sci U S A 2005; 102: 7677-82.

11. Visintin I, Feng Z, Longton G, et al. Diagnostic markers for early detection of ovarian cancer. Clin Cancer Res 2008; 14: 1065-72. Erratum in: Clin Cancer Res 2008; 14: 5308; Clin Cancer Res 2008; 14: 7158.

12. Maccio A, Madeddu C, Massa D, et al. Hemoglobin levels correlate with interleukin-6 levels in patients with advanced untreated epithelial ovarian cancer: role of inflammation in cancer-related anemia. Blood 2005; 106: 362-7.

13. Nick AM, Sood AK. The ROC ' $n$ ' role of the multiplex assay for early detection of ovarian cancer. Nat Clin Pract Oncol 2008; 5: 568-9.

14. Abramov Y, Anteby SO, Fatum M, Fasouliotis SJ, Barak V. The kinetics of leptin in Meigs' syndrome. Gynecol Oncol 2001; 83: 316-8.

15. Cvijovic G, Yamashita SA, Micic D, Kendereski A, Sumarac-Dumanovic M, Zoric S, Popovic V. Low leptin level in an obese hyperandrogenic woman - potential marker for androgen secreting tumor. Gynecol Endocrinol 2007; 23: 112-6.

16. Barber MD, McMillan DC, Wallace AM, Ross JA, Preston T, Fearon KC. The response of leptin, interleukin- 6 and fat oxidation to feeding in weight-losing patients with pancreatic cancer. Br J Cancer 2004; 90: 1129-32.

17. Keim NL, Stern JS, Havel PJ. Relation between circulating leptin concentrations and appetite during prolonged, moderate energy deficit in women. Am J Clin Nutr 1998; 68: 794-801.

18. Dubuc GR, Phinney SD, Stern JS, Havel PJ. Changes of serum leptin and endocrine and metabolic parameters after 7 days of energy restriction in men and women. Metabolism 1998; 47: 429-34.

19. Chan JL, Heist K, DePaoli AM, Veldhuis JD, Mantzoros CS. The role of falling leptin levels in the neuroendocrine and metabolic adaptation to short-term starvation in healthy men. J Clin Invest 2003; 111: 1409-21.
20. Leibel RL, Rosenbaum M, Hirsch J. Changes in energy expenditure resulting from altered body weight. N Engl J Med 1995; 332: 621-8.

21. Stattin P, Palmqvist R, Söderberg S, Biessy C, Ardnor B, Hallmans G, Kaaks R, Olsson T. Plasma leptin and colorectal cancer risk: a prospective study in Northern Sweden. Oncol Rep 2003; 10: 2015-21.

22. Stattin P, Söderberg S, Biessy C, Lenner P, Hallmans G, Kaaks R, Olsson T. Plasma leptin and breast cancer risk: a prospective study in northern Sweden. Breast Cancer Res Treat 2004; 86: 191-6.

23. Dulger H, Alici S, Sekeroglu MR, Erkog R, Ozbek H, Noyan T, Yavuz M. Serum levels of leptin and proinflammatory cytokine in patients with gastrointestinal cancer. Int J Clin Pract 2004; 58: 545-9.

24. Mantovani G, Maccio A, Mura L, et al. Serum levels of leptin and proinflammatory cytokines in patients with advanced-stage cancer at different sites. J Mol Med 2000; 78: 554-61.

25. Yedema CA, Kenemans P, Thomas CM, et al. CA 125 serum levels in the early post-operative period do not reflect tumour reduction obtained by cytoreductive surgery. Eur J Cancer 1993; 29A: 966-71.

26. Mogensen O, Brock A, Holm Nyland M. CA 125 measurements in ovarian cancer patients during their first postoperative week. Int J Gynecol Cancer 1993; 3: 54-6.

27. Gaducci A, Landoni F, Maggino T, Sartori E, Zola P, Fanucchi A. The relationship between postoperative decline of serum CA125 levels and size of residual disease after initial surgery in patients with advanced ovarian cancer: a CTF Study. Gynecol Oncol 1996; 63: 234-7.

28. Diaz ES, Karlan BY, Li AJ. Obesity-associated adipokines correlate with survival in epithelial ovarian cancer. Gynecol Oncol 2013; 129: 353-7.

\section{Address for correspondence}

Jacek Grabowski MD, PhD

Department of Gynaecology and Gynaecological Oncology Kliniken-Essen-Mitte

Henricistr. 92

45136 Essen

Germany

e-mail: jacgrab@yahoo.com

Submitted: 15.09 .2014

Accepted: 22.10 .2014 